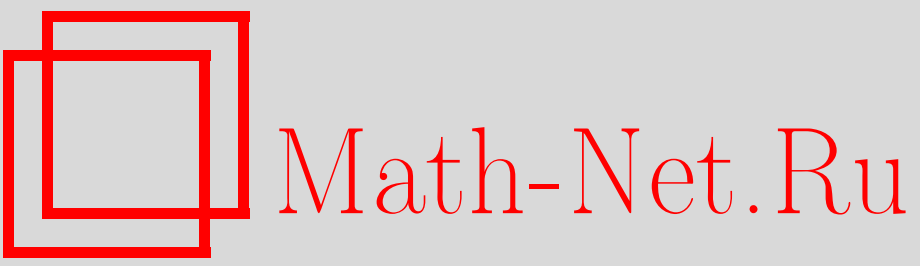

П. В. Гапеев, К доказательству Первой Фундаментальной Теоремы финансовой математики, УМH, 1998, том 53, выпуск 6, 245-246

DOI: https://doi.org/10.4213/rm98

Использование Общероссийского математического портала Math-Net.Ru подразумевает, что вы прочитали и согласны с пользовательским соглашением

http://www.mathnet.ru/rus/agreement

Параметры загрузки:

IP : 54.157 .27 .8

26 апреля 2023 г., 17:26:51 


\title{
К ДОКАЗАТЕЛЬСТВУ ПЕРВОЙ ФУНДАМЕНТАЛЬНОЙ ТЕОРЕМЫ ФИНАНСОВОЙ МАТЕМАТИКИ
}

\author{
П. В. Г АПЕЕВ
}

Как известно, Первая Фундаментальная Теорема финансовой математики представляет собой следующее утверждение: Отсутствие в модели финансового рынка некоторого условия возможности получения прибыли без риска равносильно существованию әквивалентной мартингальной мерь.

В статьях $\Phi$. Делбаена и В. Шахермайера [1], [2] содержится доказательство Теоремы в случае непрерьвного времени с использованием фактов функционального и выпуклого анализа, а именно, теорему Хана-Банаха и лемму Яна-Крепса об отделимости.

А.Н. Ширяев и Ж. Жакод в работе [3] провели наиболее полное и ясное доказательство Теоремы в модели с дискретным временем.

Цель данной работы - показать, что имеет право на существование доказательство Teоремы в случае непрерывного времени, исползующее утверждение для дискретных аппроксимаций процессов и последующий естественньй для теории вероятностей предельный переход при неограниченном измельчении разбиений временной оси.

Мы построим последовательность моделей рынков, процессы в которых являются дискретизациями процессов исходной модели с непрерывным временем. Такая последовательность, следуя терминологии статьи Ю. М. Кабанова и Д. О. Крамкова [4], является моделюю “большого рынка". Далее, мы покажем, что последовательность дискретизаций обладает свойством слабой сходимости к исходному процессу. Более того, для произвольной стратегии в модели рынка существует последовательность стратегий на построенном большом рынке, капиталы которых слабо сходятся к капиталу исходной стратегии. И наконец, мы докажем, что в случае, когда процесс дисконтированных цен финансового актива является ограниченньг семимартингалом, введенное в работе И. Кляйн [5] условие отсутствия асимптотического арбитража $\widetilde{N A A}$ (No Asymptotic Free Lunch with Bounded Risk) в построенной модели большого рьнка эквивалентно введенному в статье [1] условию безарбитражности $\widetilde{N A}($ No Free Lunch with Bounded Risk) в исходной модели рынка с непрерывньм временем.

Пусть на некотором стохастическом базисе $\mathbf{B}=\left(\Omega, \mathscr{F},(\mathscr{F})_{t \geqslant 0}, \mathrm{P}\right)$ задан семимартингал $S=\left(S_{t}\right)_{t \geqslant 0}$, порождающий фильтрацию $\mathscr{F} t=\sigma\left(S_{u}: u \in[0, t]\right)$, являющуюся непрерьвной справа и пополненной относительно меры $\mathrm{P}$.

Предположим, что $S$ выражает флуктуацию во времени цены некоторого рискового финансового актива на фондовом рьнке, выраженной в единицах другого актива, например - банковского счета, тогда капитал некоторой допустимой самофинансируемой стратегии $\pi$ имеет представление $X_{t}^{\pi}=(\gamma \bullet S)_{t} \geqslant-a, t \in \mathbb{R}_{+}$, Р-п.н., для некоторого $a \geqslant 0$, где $\gamma=\left(\gamma_{t}\right)_{t \geqslant 0}$ - предсказуемьй процесс, определяющий количество единиц актива $S$ в момент времени $t$.

Определим последовательность разбиений временной оси $\mathbb{R}_{+}: \mathscr{T}(n)=\mathscr{T}(n-1) \cup T(n)$, $T(n)=\left\{k / n \mid k=0,1, \ldots, n^{2}\right\}, k(n)$ - число точек разбиения. Для каждого разбиения $\mathscr{T}(n)$ определим дискретизацию исходного процесса цен $S^{n}=\left(S_{t}^{n}\right)_{t \geqslant 0}$ :

$$
S_{t}^{n}= \begin{cases}S_{t_{i}^{n}}, & t \in\left[t_{i}^{n}, t_{i+1}^{n}\right), t_{i}^{n} \in \mathscr{T}(n), t_{i}^{n}<t_{i+1}^{n}, i=0, \ldots, k(n)-1, \\ S_{n}, & t \in[n, \infty)\end{cases}
$$

и стохастический базис $\mathbf{B}^{n}=\left(\Omega, \mathscr{F}^{n},\left(\mathscr{F}_{t}\right)_{t \geqslant 0}^{n}, \mathrm{P}^{n}\right), \mathrm{P}^{n}=\mathrm{P} \mid \mathscr{F}^{n}$, фильтрация которого порождена процессом $S^{n}: \mathscr{F}_{t}^{n}=\sigma\left(S_{u}^{n}: u \in[0, t]\right)$, является непрерьвной справа и пополненной относительно меры $\mathrm{P}^{n}$.

Таким образом, для каждого разбиения мы определили модель рынка, в которой цены финансового актива изменяются лишь конечное число раз. Капитал допустимой самофинансируемой стратегии $\pi(n)$ в этой модели имеет вид $X_{t}^{\pi(n)}=\left(\gamma^{n} \bullet S^{n}\right)_{t} \geqslant-a, t \in \mathbb{R}_{+}$, Р-п.н., для некото- 
рого $a \geqslant 0$, где процесс $\gamma^{n}=\left(\gamma_{t}^{n}\right)_{t \geqslant 0}$ является дискретизацией исходного процесса $\gamma$ :

$$
\gamma_{t}^{n}= \begin{cases}\gamma_{0}, & t \in\left[0, t_{0}^{n}\right], \\ \gamma_{t_{i}^{n}}, & t \in\left(t_{i}^{n}, t_{i+1}^{n}\right], t_{i}^{n} \in \mathscr{T}(n), t_{i}^{n}<t_{i+1}^{n}, i=0, \ldots, k(n)-1, \\ \gamma_{n}, & t \in(n, \infty) .\end{cases}
$$

Построенная последовательность моделей рьнков, соответствующих разбиениям $\mathscr{T}(n)$, является моделью болшшого рынка, опеределенной в работе [4].

Сформулируем вспомогательные результаты, раскрывающие цель предыдущих построений.

Лемма 1. Пусть $S=\left(S_{t}\right)_{t \geqslant 0}$ - ограниченный семимартингал.

Тогда для последовательности прочессов $S^{n}=\left(S_{t}^{n}\right)_{t \geqslant 0}, n \in \mathbb{N}$, выполнено условие слабой сходимости к прочессу $S$ :

$$
\mathscr{L}\left(S^{n} \mid \mathrm{P}^{n}\right) \rightarrow \mathscr{L}(S \mid \mathrm{P}), \quad n \rightarrow \infty .
$$

Лемма 2. Для любой стратегии $\pi$ на исходном рынке В существует последовательность стратегий $\pi\left(n_{k}\right)$, определенных на рынках $\mathbf{B}^{n_{k}}$, капиталь которьх слабо сходятся к капиталу стратегии $\pi$ :

$$
\mathscr{L}\left(X^{\pi\left(n_{k}\right)}=\left(\gamma^{n_{k}} \bullet S^{n_{k}}\right) \mid \mathrm{P}^{n_{k}}\right) \rightarrow \mathscr{L}\left(X^{\pi}=(\gamma \bullet S) \mid \mathrm{P}\right), \quad k \rightarrow \infty .
$$

Сформулируем основной результат.

Теорема. Пусть $S$ - ограниченный семимартингал. Тогда условие $(\widetilde{N A})$ (No Freе Lunch with Bounded Risk) (из работы [1]) в исходной модели рынка әквивалентно условию $(\widetilde{N A A})$ (No Asymptotic Free Lunch with Bounded Risk) (из pаботы [5]) в построенной модели большого рынка.

\section{СПИСОК ЛИТЕРАТУРЫ}

[1] Delbaen F., Schachermayer W. // Math. Ann. 1994. V. 300. P. 463-520. [2] Delbaen F., Schachermayer W. The Fundamental Theorem of Asset Pricing for Unbounded Stochastic Processes // Preprint. Zürich: ETH-Zentrum, 1997. [3] Jacod J., Shiryaev A. N. // Finance Stoch. 1998. V. 2. № 3. Р. 259-273. [4] Кабанов Ю. М., Крамков Д. О. // Теория вероятн. и ее примен. 1994. Т. 39. № 1. С. 222-228. [5] Klein I. Asymptotic Arbitrage Theory // Philosophical Dissertation.

Московский государственньй

Принято редколлегией университет им. М. В. Ломоносова

02.09.1998 